\title{
Qualitative Data Collection and Analysis Methods: The INSTINCT Trial
}

William J. Meurer, MD, Shirley M. Frederiksen, MS, RN, Jennifer J. Majersik, MD, Lingling Zhang, MA, Annette Sandretto, MSN, Phillip A. Scott, MD

\begin{abstract}
Patient care practices often lag behind current scientific evidence and professional guidelines. The failure of such knowledge translation (KT) efforts may reflect inadequate assessment and management of specific barriers confronting both physicians and patients at the point of treatment level. Effective KT in this setting may benefit from the use of qualitative methods to identify and overcome these barriers. Qualitative methodology allows in-depth exploration of the barriers involved in adopting practice change and has been infrequently used in emergency medicine research. The authors describe the methodology for qualitative analysis within the INcreasing Stroke Treatment through INteractive behavioral Change Tactics (INSTINCT) trial. This includes processes for valid data collection and reliable analysis of the textual data from focus group and interview transcripts. INSTINCT is a 24-hospital, randomized, controlled study that is designed to evaluate a system-based barrier assessment and interactive educational intervention to increase appropriate tissue plasminogen activator (tPA) use in ischemic stroke. Intervention hospitals undergo baseline barrier assessment using both qualitative as well as quantitative (survey) techniques. Investigators obtain data on local barriers to tPA use, as well as information on local attitudes, knowledge, and beliefs regarding acute stroke treatment. Targeted groups at each site include emergency physicians, emergency nurses, neurologists, radiologists, and hospital administrators. Transcript analysis using NVivo7 with a predefined barrier taxonomy is described. This will provide both qualitative insight on thrombolytic use and importance of specific barrier types for each site. The qualitative findings subsequently direct the form of professional education efforts and system interventions at treatment sites.
\end{abstract}

ACADEMIC EMERGENCY MEDICINE 2007; 14:1064-1071 @ 2007 by the Society for Academic Emergency Medicine

Keywords: Health services, qualitative research, research design, guideline adherence, acute stroke, knowledge translation

$\mathrm{T}$ he typical sequence of translating new research findings into commonly accepted clinical practice begins with the publication of pilot studies, followed by larger, definitive clinical trials, with subsequent dissemination throughout the medical community. New pharmaceuticals or medical devices may then undergo evaluation by regulatory bodies (e.g., U.S. Food and Drug Administration) for efficacy and safety and, if approved, become available for general use. Postapproval studies may ensue, potentially expanding the available lit-

From the Department of Emergency Medicine (WJM, SMF, AS, PAS), Department of Neurology (WJM, JJM), and the Center for Statistical Consultation and Research (LZ), University of Michigan, Ann Arbor, MI.

Received February 28, 2007; revision received May 11, 2007; accepted May 11, 2007.

Supported by the National Institute of Neurological Disorders and Stroke RO1 NS050372 and by the University of Michigan Departments of Emergency Medicine and Neurology.

Contact for correspondence and reprints: William J. Meurer, MD; e-mail: wmeurer@med.umich.edu. erature. After compelling data or completion of multiple trials, systematic reviews are published, potentially reaching a broader audience than the original research. Ultimately, the cumulative experience for new drugs, devices, techniques, or patient care approaches may be codified into consensus statements and guidelines that are issued by professional or peer organizations, further expanding the chance of influencing practitioner behavior. Figure 1 illustrates this research-to-practice pipeline. ${ }^{1}$

\section{THE PROBLEM: KNOWLEDGE TRANSLATION FAILURE}

This idealized process of knowledge translation (KT) may result in only limited success in changing practitioner behavior, particularly when disagreement on the meaning of research data or more difficult risk-reward evaluation occurs. Interestingly, even when medical providers agree that a given therapeutic strategy is beneficial, delayed adoption can occur. A recent professional guideline for the treatment of acute otitis media in children identified patients who would be candidates for observation, as opposed to antibiotic treatment. ${ }^{2}$ A subsequent survey 


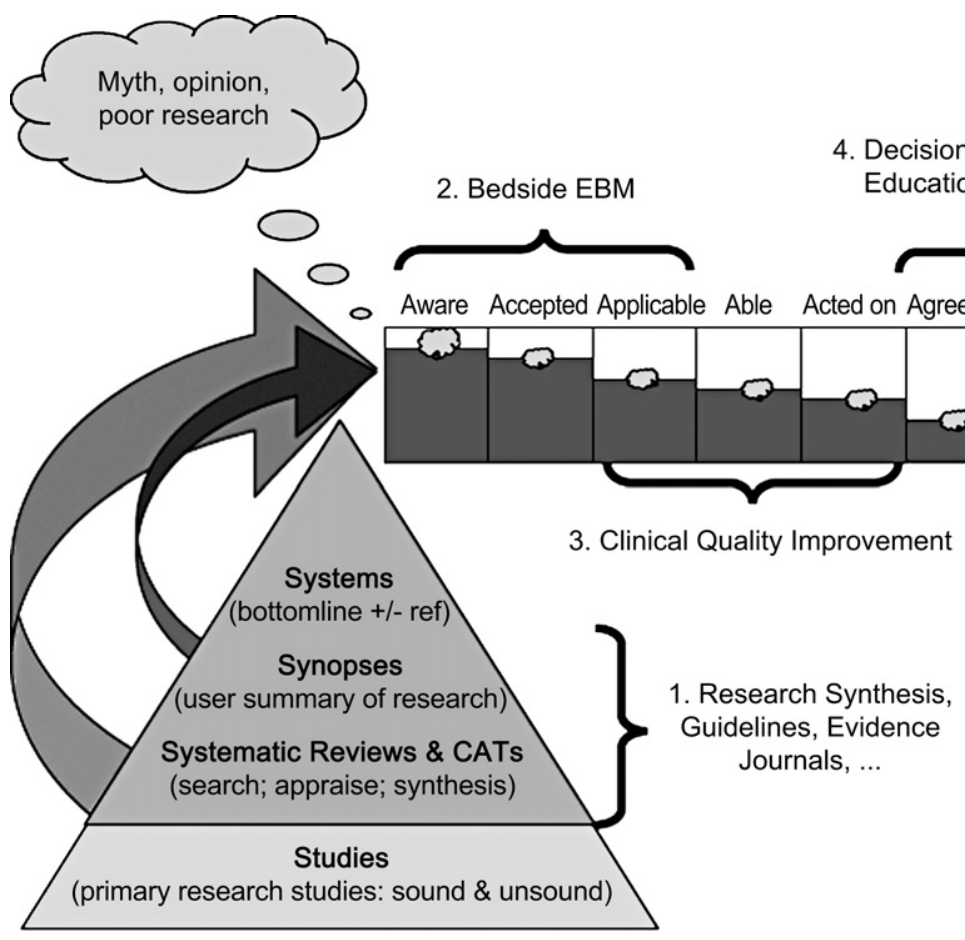

Figure 1. The research-to-practice pipeline. New research, of varying soundness, is added to the expanding pool and enters practice either directly or after first being reviewed, summarized, and systematized (delay) before entering practice. Leakage occurs at each of several stages between awareness and patient outcome. Different knowledge translation disciplines focus on different parts of the pipeline. ${ }^{1-4}$ EBM = evidence-based medicine; CATs = critical appraisal templates. Reproduced from Glasziou and Haynes, ${ }^{1}$ with permission.

indicated that $90 \%$ of physicians had read the guidelines and that $88 \%$ believed observation was a reasonable strategy. In practice, however, this management approach was used in only $10 \%$ of cases. ${ }^{3}$ This highlights the need to develop physician-targeted educational and system-based interventions that improve adoption of effective therapies.

\section{BARRIERS TO SUCCESSFUL KT}

Although health care providers possess strong internal motivation to assimilate new information to improve the quality of care that they provide, this desire must compete against numerous internal and external barriers. Simply keeping up with the literature may prove a daunting task, because the publication of randomized controlled trials is growing at an exponential rate. ${ }^{4}$ In addition, because behavioral change often must occur within a complex organization (hospital, group practice, health maintenance organization, etc.), the organization's barriers to adopting new therapies must be identified, understood, and removed for information to succeed in altering practice. Qualitative research may assist in the identification of these barriers to enhance effective KT.

\section{QUALITATIVE RESEARCH}

Qualitative research is a set of techniques, used frequently in social science and marketing, in which data are obtained from a relatively small group of respondents and are not analyzed with statistical techniques. It involves detailed, verbal descriptions of characteristics, cases, and settings, and it uses observation, interviewing, and document review as source data. It differs from traditional quantitative research through its use of less structured data-gathering instruments and its use of open-ended questioning. Results may be difficult to replicate, and the data analysis is, by nature, more subjective. Common qualitative research techniques include guided interviews, focus groups, and projective methods and allow exploration of the main dimensions of a problem while providing access to greater understanding of participant motivations as well as detail on participant behavior and attitudes.

Health services researchers have put forth proposed guidelines and position statements outlining factors that contribute to validity and rigor in this type of work. ${ }^{5-10}$ Examples cited include the so-called triangulation of data sources and methods. This process uses two or more different approaches in obtaining the data of interest and serves to increase the validity of the qualitative inquiry and ultimate robustness of the conclusions. Confirming focus group data on opinions regarding stroke treatment by using a subsequent follow-up (quantitative) survey would be one example of triangulation. Detailed tracking of the data collection and analysis process is another method to enhance the validity of the work. This allows other researchers to inspect the thought process that is involved in acquiring and analyzing the data.

\section{Use in Emergency Medicine}

Qualitative research is a relatively new technique in emergency health services research, with only minimal 
numbers of studies and detail published on the methodology and the practical aspects of collecting and analyzing qualitative data. ${ }^{11-13}$ One study of the environment of emergency medical care in Serbia used both qualitative and quantitative methods and provides a methodologically sound model for health system assessments in postconflict or postdisaster settings. ${ }^{11}$ The researchers identified critical needs in emergency medical care infrastructure and barriers to implementing them. Other qualitative work outside of emergency medicine (EM) has focused on investigations exploring the use of specific medications (i.e., aspirin in primary care ${ }^{14}$ and inhaled corticosteroids in asthma ${ }^{15,16}$ ). This work focused on describing physician behavior and attitudes relevant to clinical guideline adherence.

\section{Use in Barrier Identification}

Qualitative research approaches may provide a more complete description of barriers to adopting new procedures or therapies and offers a rational first step in designing methods to increase the rate of KT under certain conditions. This may be particularly important for enhancing clinician adoption of complex or higherrisk guidelines, such as those regarding tissue plasminogen activator (tPA) for patients with acute stroke.

This article describes the methodology for the collection and analysis of qualitative data used in the INSTINCT study (INcreasing Stroke Treatment through INteractive behavioral Change Tactics) that is described in the following section. In addition, it describes the identification, adaptation, and use of a barrier taxonomy that may be useful in qualitative research to classify barriers to KT.

\section{INSTINCT STUDY}

\section{Background}

Only $1 \%$ to $3 \%$ of stroke patients in community settings are receiving tPA therapy, 11 years after its U.S. Food and Drug Administration approval. ${ }^{17-22}$ Studies elsewhere have demonstrated that community and academic hospitals can deliver tPA effectively..$^{20,23-30}$ However, numerous internal and external barriers exist to expanding delivery more broadly in patients with stroke. ${ }^{31,32}$

Work published elsewhere found that a combination of community and professional education increased thrombolytic use in patients with stroke, from a preintervention rate of $2.2 \%$ to a postintervention rate of $11.3 \%$ (p = 0.007), with the data suggesting that the professional education component was the critical element in increasing use. ${ }^{17,33}$ This study was limited by its quasi-experimental design, single-community setting, and resource-intensive nature. ${ }^{17}$

We hypothesize that combining qualitative identification of specific barriers that physicians, staff, and hospitals face in delivering tPA to patients with acute stroke with targeted educational and corrective interventions will increase appropriate tPA use in the community setting. The institutional review boards of the University of Michigan and participating hospitals approved the protocol. Investigators in the study were certified in human subject protection and were drawn from various medical backgrounds, including EM, neurology, and internal medicine.

\section{Overview: Barrier Assessment}

The INSTINCT trial is a multicenter, randomized, controlled study that is designed to evaluate a standardized, system-based, barrier assessment and interactive educational intervention in increasing appropriate tPA use in stroke patients. The intervention targets community emergency departments (EDs) in Michigan, is based on adult education and behavior change theory, and is designed for replication in community health initiatives. It incorporates local stroke champion development, hospital- and staff-specific barrier evaluation, mixed continuing medical education that targets identified barriers, performance feedback, protocol development, and academic detailing ${ }^{34}$ (which provides individual feedback on site performance, outcomes, and education).

The primary endpoint will be an absolute increase of at least $4 \%$ in the proportion of stroke patients who are appropriately treated with intravenous tPA in intervention hospital EDs, compared with the case of matched controls. We will also measure pre- and postintervention changes in emergency physician (EP) knowledge, attitudes, and beliefs regarding tPA use in stroke in both control and treatment groups through a series of surveys.

Twenty-four hospitals were randomly selected from the population of all eligible Michigan acute care hospitals and were matched for stroke volume, hospital, and population demographics into 12 pairs. After random assignment within pairs to intervention or control arms, the 12 intervention sites undergo a two-step qualitative assessment for barriers to tPA use in stroke. The first step begins with a one-day stroke champions study initiation meeting that is attended by teams of key local physicians, nurses, and other health care providers as identified by the local site investigator. During this meeting, focus groups are conducted with all attendees.

The second barrier assessment step occurs approximately three months after the champions meeting, when a research team visits each of the 12 intervention hospitals individually and conducts an on-site qualitative barrier assessment. The research team leads two additional focus groups (one each for EPs and for nursing personnel who are not participating in the champions meeting), as well as conducts guided interviews with key representatives of the neurology, radiology, and hospital administration as identified by the local site investigator.

Barrier data obtained from these qualitative methods will be compared with additional quantitative data on barriers to tPA use that are obtained from surveys of the EPs, as well as a prespecified stroke resource assessment that is conducted by the visiting research team.

\section{Qualitative Methodology}

Discussion Guide Development. The discussion guide is a series of questions that is used by the facilitator of each focus group (or guided interview) to ensure that important themes of interest (barriers) are discussed in each session. This is of particular importance when multiple facilitators are conducting sessions simultaneously, because it increases consistency across groups and provides increased confidence that the questions obtain the specific information needed. The use of a questioning-type 
(as opposed to topic-type) discussion guide was intentional to allow more efficient analysis, because small differences in questioning could inadvertently alter the intent of the question and, thus, the response of the participants. $^{35}$

The discussion guide or script was developed with an external, professional, focus group consultant. The guidebook incorporated various categories of questions, including opening, introductory, transition, key, and ending questions. Unplanned probe and follow-up questions were specifically encouraged. In addition to discussion and question cues, the guide also provided time estimates for each group of questions to allow efficient completion of all portions while providing adequate time for group comments.

Initial versions of the discussion guides for the focus groups and guided interviews were developed to identify presumed barriers to tPA use, as well as to explore individual beliefs and attitudes toward stroke treatment. The focus group facilitators met, reviewed, and revised the guide before the champions meeting. After completion of the champions meeting, they met again and discussed each of the guide questions. Deletions of less useful questions and reorganization of the discussion guide occurred, and a final draft was developed for use at the future on-site focus groups. Thus, the champions meeting served as a pilot test for the final on-site EP and nursing discussion guide. Selected excerpts from the focus group discussions guide used for the champions meeting are available as an online Data Supplement (at http:// www.aemj.org/cgi/content/full/j.aem.2007.05.005/DC1). In addition, based on the comments regarding barriers from the champions meeting participants, we modified the discussion guides to be used for interviews with radiologists, neurologists, and administrators. For example, commonly discussed barriers regarding computed tomography scanning and interpretation provided additional questions for on-site interviews with radiologists.

Facilitator Recruitment and Training. Of the six facilitators for the champions meeting focus groups, four were research coordinators (two each from nursing and public health/social work), and two were physicians. Thus, they mirrored the professional occupations and experience of the planned participants. Three facilitators had previous experience conducting focus group discussions. In addition, a professional focus group facilitator provided a single training session in qualitative methodology and moderating techniques before the start of the study. Of the six facilitators, two were male; all six had a white racial phenotype, and their ages ranged between 30 and 60 years, allowing diverse options for paring with specific groups to enhance rapport.

\section{Data Collection}

Champions Meeting Focus Groups. The champions meeting focus groups were composed of the site investigators or physician stroke champions from each of the 12 treatment sites as well as other key personnel, as described in Overview: Barrier Assessment. Six groups ran concurrently, and each consisted of between four and six individuals. Ground rules were presented, and 90 minutes of discussion followed using the guides described in Discussion Guide Development. Responses for each group were recorded by using two digital audio recording devices to protect against inadvertent data loss.

Site Focus Groups. Two on-site focus groups (one of EPs and a separate one of emergency nursing and ancillary staff) will be performed by either study nurse coordinators or one of the investigators, trained as described in Facilitator Recruitment and Training. The revised discussion guide (from the champions meeting) as well as similar audio recording and transcription methodology will be used for all sites.

Site Guided Interviews. Guided interviews are a common qualitative, one-on-one, data collection technique. This method allows for focused exploration on individual perceptions of events relating to a limited number of topics or themes. These interviews again use open-ended questioning and have limited structure. The process of data collection is more intimate than that in focus groups. An example of the proposed script for an interview with a neurologist is presented in Figure 2. The on-site assessments include interviews with local neurologists, radiologists, and hospital administrators. We will endeavor to

1. Could you tell us a bit about your practice (number of physicians, key interest areas, whether you admit to your own service or serve solely as a consult service)?

2. How many stroke patients do you see here in a year? What type of roles does neurology have?

3. How do you see the current stroke program as it relates to your institution's strategic mission? (Prompts: Would you say it's a key component? Is this a program the institution wants to grow, maintain, or reduce?)

4. What would you see as the major strengths of your stroke program?

5. The weaknesses?

6. Do you plan to obtain JCAHO certification?

7. Have you had personal experience with giving tPA? How was it?

8. What systems here really seem to be helpful in facilitating tPA treatment?

9. What do you see as barriers to tPA treatment here? (Prompt if needed: EMS? ED? Neurology? Radiology? ICU bed availability? Diversion status in ED? Lack of neurosurgery consultation available? Data on effectiveness is not convincing? Rate of hemorrhage is unacceptable? Patients arriving too late? Refusals?)

10. What could be done to increase tPA treatment rates here?

Figure 2. Examples of questions from on-site neurologist interview. Questions in bold will be analyzed by using prespecified taxonomy. JCAHO = Joint Commission on Accreditation of Healthcare Organizations; ICU $=$ intensive care units. 
conduct all interviews in person; however, to accommodate interviewee schedules, and because of travel distances involved, telephone interviews are permitted.

Transcription. Audio recordings first undergo postprocessing to remove extraneous background noise and are transcribed. The recordings and transcriptions of individual groups are reviewed with the individual facilitator to ensure accuracy and proper assignment of comments to participants. Participant confidentiality is preserved in transcripts by coding of all names and identifiers. These codes, however, allow analysis by site (hospital), participant role (nurse, physician, or other), and specialty.

\section{Qualitative Data Analysis}

Template Codebook Development. The knowledge-attitudes-behavior framework ${ }^{36}$ provides a general scheme that can be used to characterize physician barriers to adopting a new therapy or adhering to a clinical guideline. Cabana et al. investigated this in detail by using a partial grounded theory approach to create a model that more precisely characterized barriers. ${ }^{37}$ Grounded theory is a method by which qualitative data is coded into themes. In contrast to most quantitative research, grounded theory is inductive: the data are used to form the theory, as opposed to being used to prove or refute a hypothesis. The Cabana et al. ${ }^{37}$ guideline adherence research explored the various reasons that clinicians do not adhere to practice guidelines and provided a framework for improvement. The INSTINCT trial has adapted this taxonomy of barriers ${ }^{37}$ (Figure 3 ) to code the obtained qualitative data into themes.

The use of a pre-existing taxonomy for analysis allows us to determine the importance of relationships between barriers to tPA use. The primary weakness of this approach is the assumption that identified barriers will fall into these categories. This potentially introduces bias against barriers that may emerge but do not fit into this model. Although coders are instructed to follow the predefined taxonomy, they are encouraged to keep memos and annotations, which are subsequently used to attribute ideas and themes from the transcripts into categories of barriers that were not initially available.
Coding Responses. After transcription, the data will be imported into NVivo 7, a qualitative analysis software package (QSR International Pty Ltd., Doncaster, Australia). Use of computer-assisted techniques improve procedure standardization, potentially increases efficiency, enhances completeness, and permits greater flexibility in revising the analysis process.

Champions Meeting Data. Two physician investigators will independently review and assign text segments and statements to specific barrier codes by using a template approach for data from the champions meeting. A template approach involves assigning portions of text and comments into barrier categories that are contained within a codebook that has been obtained from previous research and theoretical perspectives. Specific development of the INSTINCT codebook is described in Template Codebook Development.

After completing the initial focus group data, the physician coders will meet once to discuss the findings and whether to revise the codebook to incorporate additional barriers. If new categories are introduced, the existing assignment of textual data (participant responses from focus groups) in the database will be refined to reflect these changes. Interrater reliability of the textual coding will be determined. If interrater agreement is not found at a prespecified level of $80 \%$, the coders will be retrained and the coding of the disputed data repeated. If agreement about specific areas of text cannot be reached, a third investigator will adjudicate.

Site Focus Groups and Guided Interviews. Analysis of the subsequent on-site focus groups will be divided between the two coders and proceed independently. In the analysis of the guided interviews, responses to the questions regarding barriers will be incorporated into the qualitative database and coded according to the above taxonomy. Other aspects of these interviews will pertain to systems-based issues and be analyzed separately.

After data coding is completed, characteristics of the hospitals will be incorporated into the qualitative

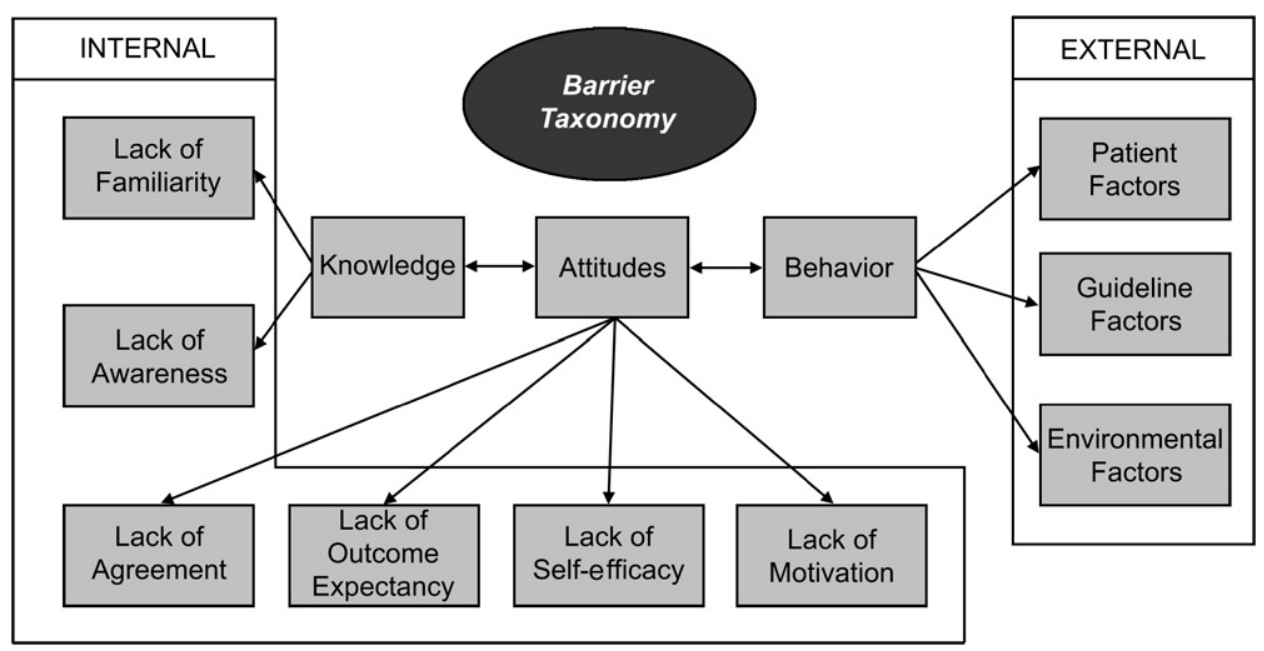

Figure 3. Taxonomy of barriers to behavior change. Adapted from Cabana et al. ${ }^{37}$ 


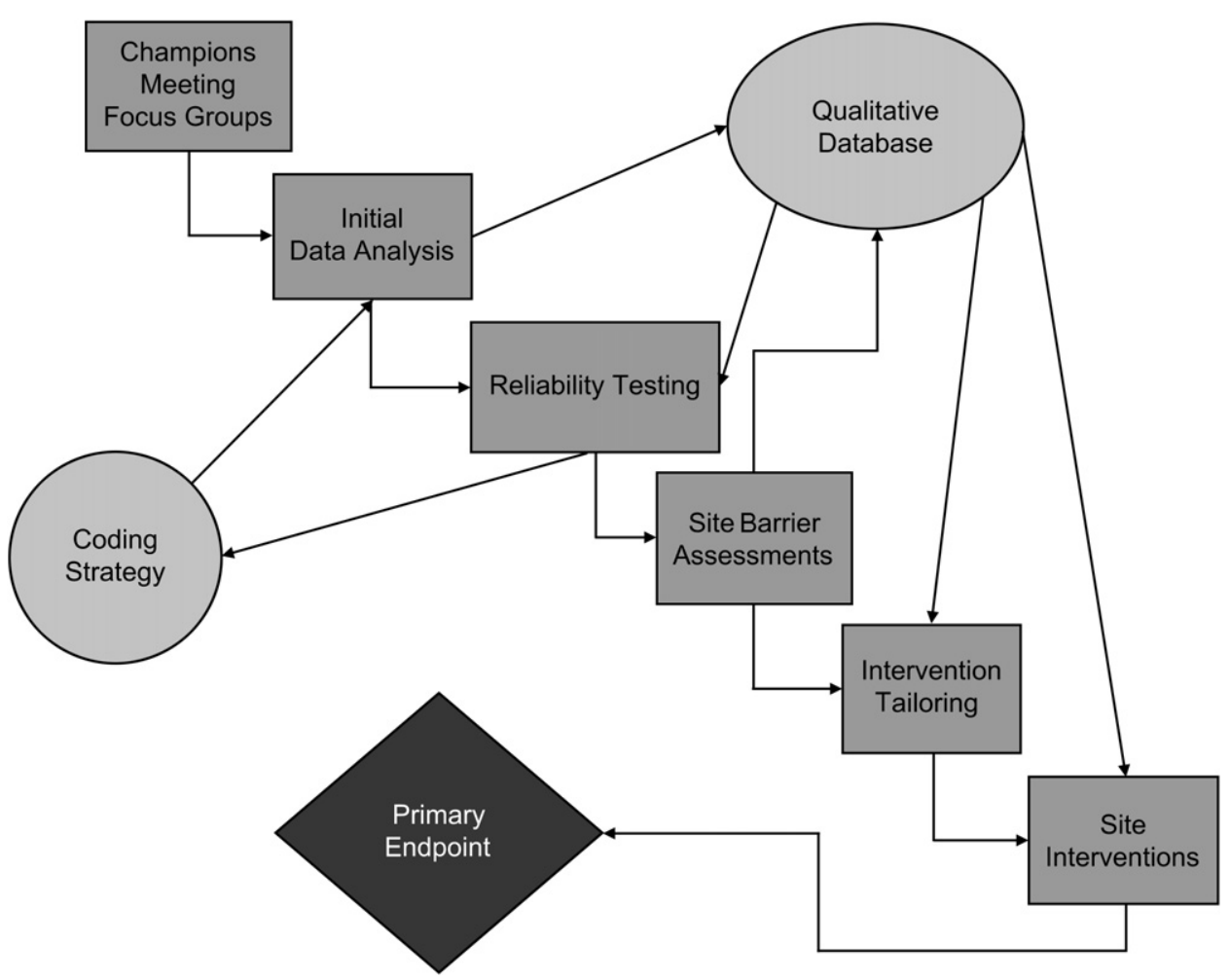

Figure 4. Data process flowchart.

database. This will allow analysis of how specific hospital characteristics, such as ED volume, inpatient census, and level of urbanization of surrounding community relate to the barriers.

\section{RESULTS}

All qualitative data will be incorporated into a single database. By using queries and modeling tools, the NVivo software provides site-specific data on frequencies of barriers and the relationship between barriers and site characteristics. Content that emerges as illustrative of the perceptions of the barriers and that provides targets for educational interventions will be identified. Queries will also be conducted to compare barriers identified at different hospital sites, as well as barriers expressed by participants with different roles. Comparing the use of quantitative survey results obtained from EPs at all sites with the qualitative results enhances the validity of the study in thoroughly identifying, exploring, and understanding the barriers.

The qualitative information obtained will be used to tailor the educational interventions, particularly CME content, to the specific needs of each site. An overview of the qualitative data collection, analysis, and application process for the INSTINCT project is presented in Figure 4.

\section{LIMITATIONS}

An important limitation of this work is that it is a research plan. The data collection and data analysis from this project are ongoing. The scheduled completion date is September 2007.

\section{CONCLUSIONS}

Qualitative research exists on a spectrum of scientific investigations: on one end, qualitative research seeks to explore meanings and opinions and to generate hypotheses regarding decision-making and behavior by using inductive reasoning. On the other end, quantitative studies test hypotheses by using objective, measurable processes and deductive logic. The INSTINCT study provides a conceptual framework and strategy to construct a qualitative data collection and analysis plan for examining barriers, attitudes, and beliefs toward the adoption of a challenging therapy in EM. It then uses these results to guide a subsequent educational intervention, which is then tested for efficacy using traditional quantitative methods. By pairing such processes, qualitative research may offer an important adjunctive tool for helping translate knowledge from clinical trials and other scientific inquiry into broadly accepted clinical practice.

\section{References}

1. Glasziou P, Haynes B. The paths from research to improved health outcomes. ACP J Club. 2005; 142(2): A8-10.

2. American Academy of Pediatrics Subcommittee on Management of Acute Otitis Media. Diagnosis and management of acute otitis media. Pediatrics. 2004; 113:1451-65.

3. Vernacchio L, Vezina RM, Mitchell AA. Knowledge and practices relating to the 2004 acute otitis media 
clinical practice guideline: a survey of practicing physicians. Pediatr Infect Dis J. 2006; 25:385-9.

4. Tsay MY, Yang YH. Bibliometric analysis of the literature of randomized controlled trials. J Med Libr Assoc. 2005; 93:450-8.

5. Devers KJ. How will we know "good" qualitative research when we see it? Beginning the dialogue in health services research. Health Serv Res. 1999; 34: 1153-88.

6. Patton MQ. Enhancing the quality and credibility of qualitative analysis. Health Serv Res. 1999; 34: 1189-208.

7. Shortell SM. The emergence of qualitative methods in health services research. Health Serv Res. 1999; 34: 1083-90.

8. Sofaer S. Qualitative methods: what are they and why use them? Health Serv Res. 1999; 34:1101-18.

9. Giacomini MK, Cook DJ. Users' guides to the medical literature: XXIII. Qualitative research in health care, B. What are the results and how do they help me care for my patients? Evidence-Based Medicine Working Group. JAMA. 2000; 284:478-82.

10. Giacomini MK, Cook DJ. Users' guides to the medical literature: XXIII. Qualitative research in health care, A. Are the results of the study valid? EvidenceBased Medicine Working Group. JAMA. 2000; 284: $357-62$.

11. Nelson BD, Dierberg K, Scepanovic M, et al. Integrating quantitative and qualitative methodologies for the assessment of health care systems: emergency medicine in post-conflict Serbia. BMC Health Serv Res. 2005; 5:14.

12. Farmer SA, Higginson IJ. Chest pain: physician perceptions and decision-making in a London emergency department. Ann Emerg Med. 2006; 48: 77-85.

13. Thurgur L, Bandiera G, Lee S, Tiberius R. What do emergency medicine learners want from their teachers? A multicenter focus group analysis. Acad Emerg Med. 2005; 12:856-61.

14. Short D, Frischer M, Bashford J, Ashcroft D. Why are eligible patients not prescribed aspirin in primary care? A qualitative study indicating measures for improvement. BMC Fam Pract. 2003. Available at: http://www.biomedcentral.com/1471-2296/4/9. Accessed May 21, 2007.

15. Cabana MD, Ebel BE, Cooper-Patrick L, Powe NR, Rubin HR, Rand CS. Barriers pediatricians face when using asthma practice guidelines. Arch Pediatr Adolesc Med. 2000; 154:685-93.

16. Scarfone RJ, Zorc JJ, Angsuco CJ. Emergency physicians' prescribing of asthma controller medications. Pediatrics. 2006; 117:821-7.

17. Morgenstern LB, Staub L, Chan W, et al. Improving delivery of acute stroke therapy: the TLL Temple Foundation Stroke Project. Stroke. 2002; 33: 160-6.

18. Chiu D, Krieger D, Villar-Cordova C, et al. Intravenous tissue plasminogen activator for acute ischemic stroke: feasibility, safety, and efficacy in the first year of clinical practice. Stroke. 1998; 29:18-22.

19. Katzan IL, Furlan AJ, Lloyd LE, et al. Use of tissuetype plasminogen activator for acute ischemic stroke: the Cleveland area experience. JAMA. 2000; 283: $1151-8$.

20. Chapman KM, Woolfenden AR, Graeb D, et al. Intravenous tissue plasminogen activator for acute ischemic stroke: a Canadian hospital's experience. Stroke. 2000; 31:2920-4.

21. Reed SD, Cramer SC, Blough DK, Meyer K, Jarvik JG, Wang DZ. Treatment with tissue plasminogen activator and inpatient mortality rates for patients with ischemic stroke treated in community hospitals. Stroke. 2001; 32:1832-40.

22. Reeves MJ, Arora S, Broderick JP, et al. Paul Coverdell Prototype Registries Writing Group. Acute stroke care in the US: results from 4 pilot prototypes of the Paul Coverdell National Acute Stroke Registry. Stroke. 2005; 36:1232-40.

23. Davenport J, Hanson SK, Altafullah IM, et al. tPA: a rural network experience. Stroke. 2000; 31:1457-8.

24. Grond M, Stenzel C, Schmulling S, et al. Early intravenous thrombolysis for acute ischemic stroke in a community-based approach. Stroke. 1998; 29:1544-9.

25. Katzan IL, Sila CA, Furlan AJ. Community use of intravenous tissue plasminogen activator for acute stroke: results of the brain matters stroke management survey. Stroke. 2001; 32:861-5.

26. Wang DZ, Rose JA, Honings DS, Garwacki DJ, Milbrandt JC. Treating acute stroke patients with intravenous tPA. The OSF stroke network experience. Stroke. 2000; 31:77-81.

27. Tanne D, Bates VE, Verro P, et al. Initial clinical experience with IV tissue plasminogen activator for acute ischemic stroke: a multicenter survey. The t-PA Stroke Survey Group. Neurology. 1999; 53(2):424-7.

28. Albers GW, Bates VE, Clark WM, Bell R, Verro P, Hamilton SA. Intravenous tissue-type plasminogen activator for treatment of acute stroke: the Standard Treatment with Alteplase to Reverse Stroke (STARS) study. JAMA. 2000; 283:1145-50.

29. Smith RW, Scott PA, Grant RJ, Chudnofsky CR, Frederiksen SM. Emergency physician treatment of acute stroke with recombinant tissue plasminogen activator: a retrospective analysis. Acad Emerg Med. 1999; 6:618-25.

30. Scott P, Davis L, Frederiksen S, Smith R. Emergency physician administration of rt-PA in acute stroke: five-year analysis of treatment and outcome [abstract]. Acad Emerg Med. 2002; 9:447.

31. Alberts MJ. tPA in acute ischemic stroke: United States experience and issues for the future. Neurology. 1998; 51(3 Suppl. 3):S53-5.

32. Rapid Identification and Treatment of Acute Stroke. In: Marler JR, Winters Jones P, Marian EMR (eds). Proceedings of a National Symposium on Rapid Identification and Treatment of Acute Stroke. Bethesda, MD: National Institute of Neurological Disorders and Stroke, 1997. Available at: http://www.ninds.nih. gov/news_and_events/proceedings/strokeworkshop. htm. Accessed Jul 2, 2007.

33. Morgenstern LB, Bartholomew LK, Grotta JC, Staub L, King M, Chan W. Sustained benefit of a community and professional intervention to increase acute stroke therapy. Arch Intern Med. 2003; 163: 2198-202. 
34. Soumerai SB, Avorn J. Principles of educational outreach ("academic detailing") to improve clinical decision making. JAMA. 1990; 263:549-56.

35. Morgan DL, Krueger RA, King JA. The Focus Group Kit, Volume 3: Developing Questions for Focus Groups. Thousand Oaks, CA: Sage, 1998, pp 12-3.
36. Woolf SH. Practice guidelines: a new reality in medicine. III. Impact on patient care. Arch Intern Med. 1993; 153:2646-55.

37. Cabana MD, Rand CS, Powe NR, et al. Why don't physicians follow clinical practice guidelines? A framework for improvement. JAMA. 1999; 282:1458-65. 